\title{
Mirizzi's syndrome in Roux-en-Y bypass patient successfully treated with cholangioscopically-guided laser lithotripsy via percutaneous gastrostomy
}

\section{(ㄷ)(1) $\odot($}

\author{
Authors \\ Stanislas Chaussade ${ }^{1,2}$, Frédéric Prat ${ }^{1,2}$ \\ Institutions \\ 1 Service de gastroentérologie et endoscopie digestive, \\ Hôpital Cochin, Université Paris Descartes, 27 rue du \\ Faubourg Saint Jacques, 75014 Paris, France \\ 2 Unité INSERM 1016, Université Paris Descartes, \\ Sorbonne Paris Cité, Paris, France
}

Nassim Hammoudi ${ }^{1}$, Bertrand Brieau ${ }^{1}$, Maximilien Barret ${ }^{1,2}$, Benoit Bordacahar ${ }^{1}$, Sarah Leblanc ${ }^{1}$, Romain Coriat ${ }^{1,2}$,

submitted 29.10.2017

accepted after revision 13.3 .2018

\author{
Bibliography \\ DOI https://doi.org/10.1055/a-0609-6448 | \\ Endoscopy International Open 2018; 06: E826-E829 \\ (c) Georg Thieme Verlag KG Stuttgart · New York \\ ISSN 2364-3722
}

Corresponding author

Bertrand Brieau, MD, Service de gastroentérologie et endoscopie digestive, Hôpital Cochin, Université Paris

Descartes, 27 rue du Faubourg Saint Jacques, 75014 Paris, France

Fax: +33-1-58411930

bertrand.brieau@aphp.fr

\section{ABSTRACT}

Obesity and bariatric surgery are major risk factors in gallstone disease. In patients with a past history of Roux-en-Y gastric bypass, Mirizzi's syndrome is a challenging endoscopic situation because of the modified anatomy. Here we report the first case of a patient with a Roux-en-Y gastric bypass treated by intracorporeal lithotripsy with a digital single-operator cholangioscope following an endoscopic retrograde cholangiopancreatography (ERCP) using a percutaneous gastrostomy access.

\section{Introduction}

Obesity and weight loss are associated with an increased risk of gallstone disease. Obesity doubles the risk of gallstone disease [1], and $30 \%$ to $50 \%$ of bariatric surgery patients [2,3] develop cholelithiasis, with bile cholesterol hypersaturation resulting from rapid weight loss, one of the main factors inducing a highly lithogenic state [2]. Mirizzi's syndrome (MS) is a particular complication of gallstone disease defined as a complete or partial obstruction of the common hepatic duct induced by a stone impacted in the cystic duct or the neck of the gallbladder [4]. The incidence of MS is low, varying from $0.7 \%$ to $1.4 \%$ in patients undergoing biliary surgery [4]. Treatment consists of gallstone extraction, and whenever possible, an endoscopic approach is preferred, especially in patients with a history of cholecystectomy [5]. Because stones have not fully passed through the common bile duct, endoscopic extraction can be challenging. In the case of bypass surgery, changes to the digestive anatomy further increase difficulties by complicating biliary access.

Here, we report the case of a patient with a past history of cholecystectomy and Roux-en-Y bypass, diagnosed with MS, and treated using endoscopic modalities including cholangioscope-assisted intracorporeal lithotripsy.

\section{Case report}

In December 2016, a 59-year-old woman was admitted to A\&E with abdominal pain, fever, and jaundice. She had a medical history of cholecystectomy for acute cholecystitis in 2012 and bariatric surgery with Roux-en-Y gastric bypass in 2013, resulting in a weight loss of $25 \mathrm{~kg}$. Blood tests showed hepatic cholestasis and inflammatory syndrome (C reactive protein: $103 \mathrm{mg} / \mathrm{L}$, leukocytes: $13450 / \mathrm{mm}^{3}$ ). Abdominal ultrasonography disclosed intra- and extrahepatic bile duct dilatation. As cholangitis was suspected, the patient received intravenous antibiotics, analgesics, and antipyretics. In order to allow an endoscopic ap- 


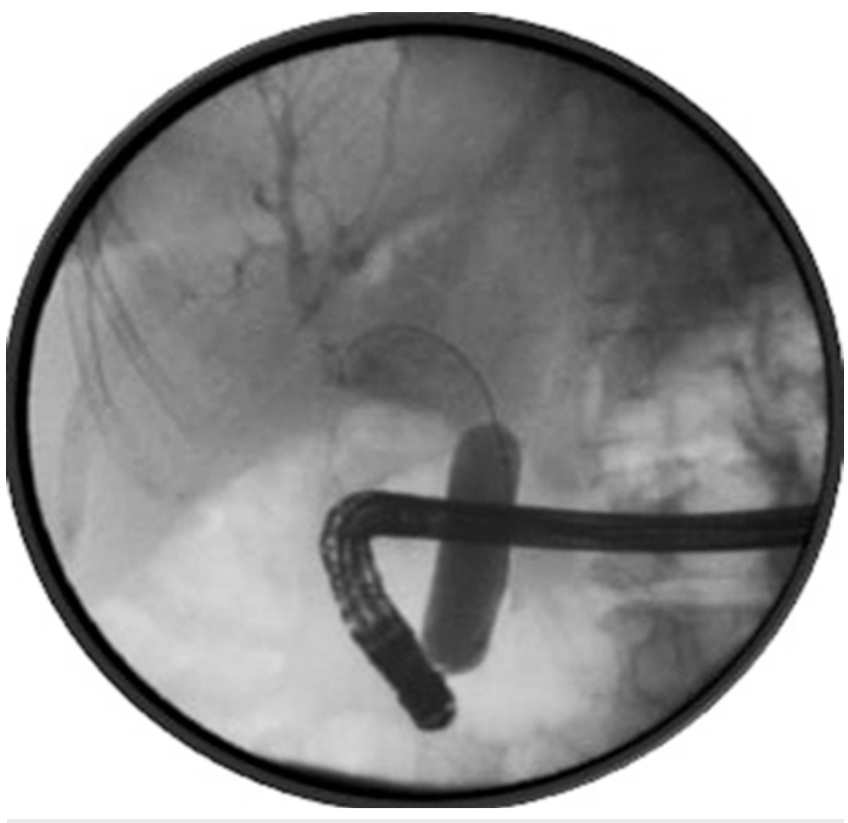

- Fig. 1 Dilation of the common bile duct to $18 \mathrm{~mm}$ using a CRE balloon.

proach to the papilla and eventually relieve biliary obstruction, a surgical laparoscopic gastrostomy of the excluded stomach was performed uneventfully.

Endoscopic retrograde cholangiopancreatography (ERCP) was subsequently performed 1 week after the patient's admission. After an easy progression through the excluded stomach and duodenum, the papilla was located next to a small diverticulum. Retrograde contrast injection revealed a contrast defect with distal bile duct stenosis suggestive of a large obstructive gallstone in a long and broad cystic stump located $2 \mathrm{~cm}$ upstream of the papilla. This clogged the common bile duct, which was mildly dilated upstream at $12 \mathrm{~mm}$, along with enlarged intrahepatic bile ducts, leading to the diagnosis of MS.

An endoscopic sphincterotomy was carried out uneventfully, but stone extraction failed due to stone size (about $3 \mathrm{~cm}$ ) and impaction within the cystic stump. A plastic stent was placed in the main bile duct to relieve jaundice, and the patient was referred to our unit for further attempts to clear the bile ducts using intracorporeal laser lithotripsy.

After removal of the stent, considering the relatively narrow distal bile duct, we first performed hydrostatic balloon dilation up to $18 \mathrm{~mm}$ ( $\triangleright$ Fig. 1), but stone mobilization remained unsuccessful. A Spyglass catheter (Boston Scientific, Marlborough, MA, United States) was subsequently inserted through the duodenoscope (Olympus TJF160, Tokyo, Japan) allowing direct visualization of the impacted gallstone. Lithotripsy was carried out using a Holmium laser (Auriga QI, Boston Scientific) at a power of $800 \mathrm{~mJ}$, then $1200 \mathrm{~mJ}$, and a frequency of $4 \mathrm{~Hz}$, then $6 \mathrm{~Hz}$ ( $\triangleright$ Fig. 2). Intracorporeal lithotripsy lasted for 25 minutes and permitted step-by-step fragmentation of the stone allowing sequential extraction of stone pieces using a Dormia basket and a balloon catheter ( $\triangleright$ Fig.3). At the end of the procedure, the main biliary tract and the cystic stump were completely

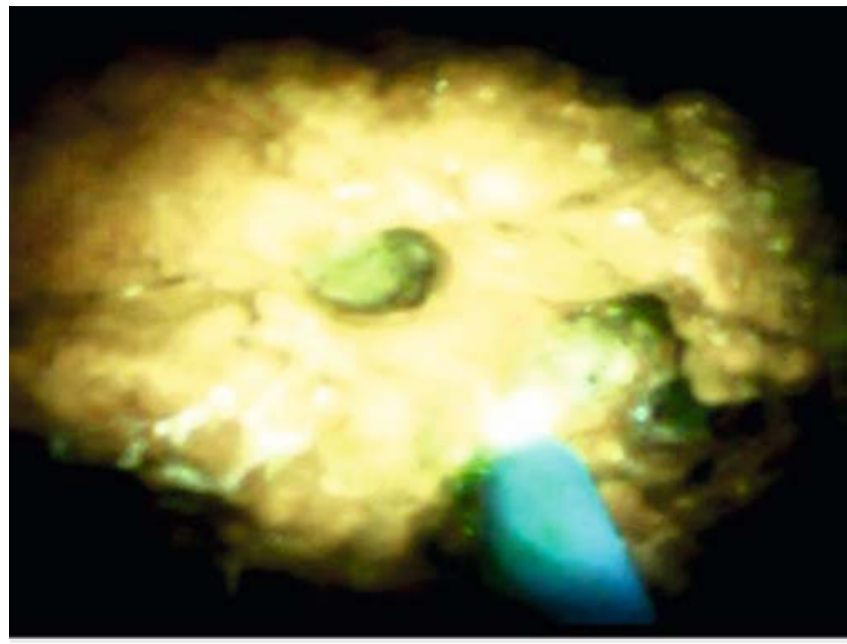

Fig. 2 Lithotripsy of the large gallstone using a Holmium laser

empty of any stone residue ( $>$ Fig. 4 ) and biliary drainage was complete with diffuse pneumobilia.

Eventually, bilirubin level and liver tests returned to normal values, jaundice disappeared, and the patient was discharged 4 days after lithotripsy. An abdominal CT scan was performed 2 months after lithotripsy showing no abnormalities and allowing closure of the gastrostomy shortly thereafter.

\section{Discussion}

We report a successful treatment of a complicated biliary stone requiring advanced endoscopic techniques including intracorporeal lithotripsy and cholangioscopy in the context of anatomic changes induced by bariatric surgery. Although challenging, endoluminal solutions in cases of bypass surgery remain possible even though the traditional per-oral endoscopic approach is not feasible. Endoscopic options in such patients include ERCP via percutaneous gastrostomy (GERCP) and ERCP via double balloon enteroscopy (DBERCP). These two techniques were compared in a study by Choi and colleagues [6] in 72 patients undergoing ERCP in the context of Roux-en-Y gastric bypass. GERCP was more effective to access the pancreatobiliary tree with a $97 \%$ success rate compared with $78 \%$ in the DBERCP group. GERCP was also a shorter procedure. Although a slight increase in complications ( $14.5 \%$ in that group) was related to the gastrostomy, side effects were mostly minor, and did not affect the patients' prognosis or interfere with the success of the endoscopic procedures.

More recently, a systematic review of 509 patients who had undergone a transgastric ERCP in Roux-en-Y gastric bypass [7] reported successful gastric access in $100 \%$ of patients and successful ductal cannulation in $98.5 \%$. Adverse events were reported in $14 \%$ of patients, $80 \%$ of which were related to the gastrostomy. Thus, and unless further data support the use of innovative methods such as EUS-guided Lumen Apposition Metallic Stents to create transgastric anastomosis [8], we consider it reasonable to recommend GERCP rather than DBERCP, all the more so because the latter offers limited interventional possibi- 


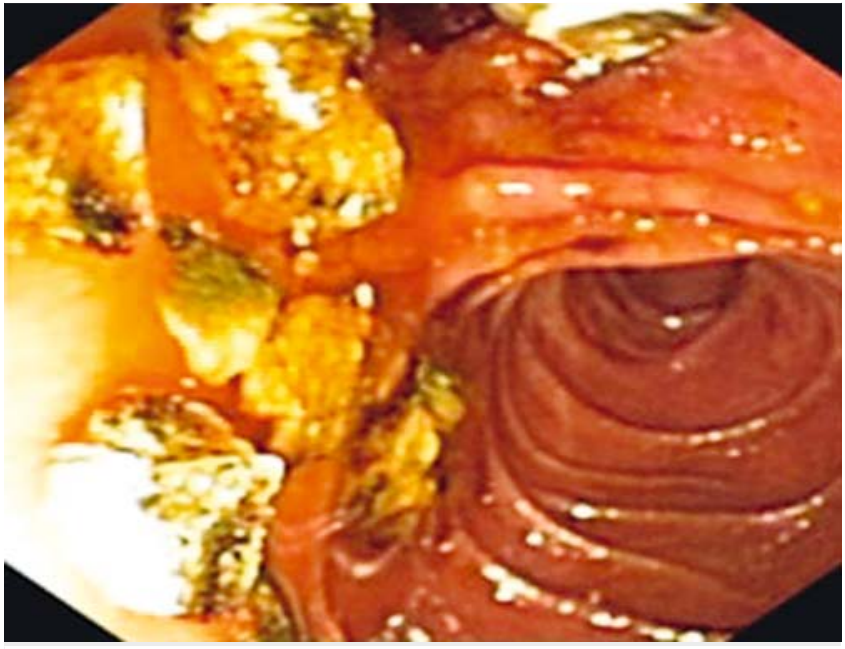

Fig. 3 Sequential extraction of the gallstone using a Dormia basket and then a balloon catheter.

lities related to the type of endoscope used. On the other hand, GERCP offers the full range of technical capabilities of ERCP, including single-operator cholangioscopy.

Cholangioscopy-directed lithotripsy has been used successfully for the treatment of difficult bile stones since the $1990 \mathrm{~s}$ [9], with success rates ranging from $74 \%$ to $98 \%$ and has been found to be safe at long-term follow-up [10]. The recently developed digital single-operator per-oral cholangioscope (D$\mathrm{SOC}$ ) offers high-resolution digital imaging, allowing superior visualization of the bile ducts compared to an earlier generation device. D-SOC was recently studied in 17 patients having complicated biliary stones. All patients had an impacted biliary stone either greater than $1.5 \mathrm{~cm}$ in diameter or which had failed capture or crushing by mechanical lithotripsy. A 94\% stone clearance was obtained after a median of 1 procedure using a Holmium laser lithotripsy, with no major adverse events [11].

In Mirizzi's syndrome, this technique has previously been described in a series of 31 patients using Spyglass technology and Holmium laser with energy levels set at 800 to $1500 \mathrm{~mJ}$ at a frequency of 8 to $15 \mathrm{~Hz}$ with a success rate of $100 \%$ after one single session [12].

Our case is the first to describe a lithotripsy procedure for bile duct stone with GERCP, illustrating that a combination of two advanced techniques can help to solve complex situations.

In conclusion, combining advanced endoscopic techniques to treat a complicated gallstone disease is possible, and bariatric surgery such as Roux-en-Y bypass does not represent a contraindication anymore, even in the case of a large gallstone requiring laser lithotripsy.

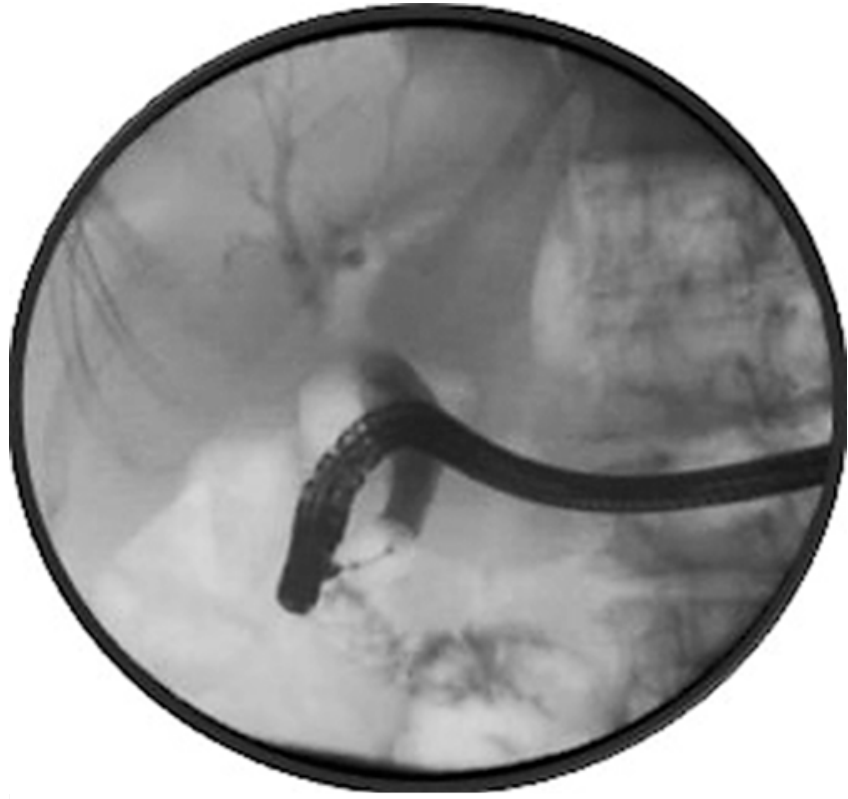

- Fig. 4 Main biliary tract and cystic stump completely empty of any stone residue. We noted the duodenoscope position from the left side through the gastrostomy.

Competing interests

None

References

[1] Friedman GD, Kannel WB, Dawber TR. The epidemiology of gallbladder disease: Observations in the Framingham Study. J Chronic Dis 1966; 19: 273-292

[2] Everhart JE. Contributions of obesity and weight loss to gallstone disease. Ann Intern Med 1993; 119: 1029-1035

[3] Shiffman ML, Sugerman H], Kellum JM et al. Gallstone formation after rapid weight loss: a prospective study in patients undergoing gastric bypass surgery for treatment of morbid obesity. Am J Gastroenterol 1991; 86: 1000 - 1005

[4] Abou-Saif A, Al-Kawas FH. Complications of gallstone disease: Mirizzi syndrome, cholecystocholedochal fistula, and gallstone ileus. Am J Gastroenterol 2002; 97: 249-254

[5] Trikudanathan G, Arain MA, Attam R et al. Advances in the endoscopic management of common bile duct stones. Nat Rev Gastroenterol Hepatol 2014; 11: 535 - 544

[6] Choi EK, Chiorean MV, Coté GA et al. ERCP via gastrostomy vs. double balloon enteroscopy in patients with prior bariatric Roux-en-Y gastric bypass surgery. Surg Endosc 2013; 27: 2894-2899

[7] Banerjee N, Parepally M, Byrne TK et al. Systematic review of transgastric ERCP in Roux-en-Y gastric bypass patients. Surg Obes Relat Dis 2017; $13: 1236-1242$

[8] Tyberg A, Nieto J, Salgado S et al. Endoscopic ultrasound (EUS)-directed transgastric endoscopic retrograde cholangiopancreatography or EUS: mid-term analysis of an emerging procedure. Clin Endosc 2017; 50: $185-190$ 
[9] Prat F, Fritsch J, Choury AD et al. Laser lithotripsy of difficult biliary stones. Gastrointest Endosc 1994; 40: $290-295$

[10] Tsuyuguchi T, Sakai Y, Sugiyama $\mathrm{H}$ et al. Long-term follow-up after peroral cholangioscopy-directed lithotripsy in patients with difficult bile duct stones, including Mirizzi syndrome: an analysis of risk factors predicting stone recurrence. Surg Endosc 2011; 25: 2179-2185
[11] Wong JC, Tang RS, Teoh AY et al. Efficacy and safety of novel digital single-operator peroral cholangioscopy-guided laser lithotripsy for complicated biliary stones. Endosc Int Open 2017; 5: E54 -E58

[12] Bhandari S, Bathini R, Sharma A et al. Usefulness of single-operator cholangioscopy-guided laser lithotripsy in patients with Mirizzi syndrome and cystic duct stones: experience at a tertiary care center. Gastrointest Endosc 2016; 84: 56-61 